\title{
OBITUARY: Joseph Leonard Doob
}

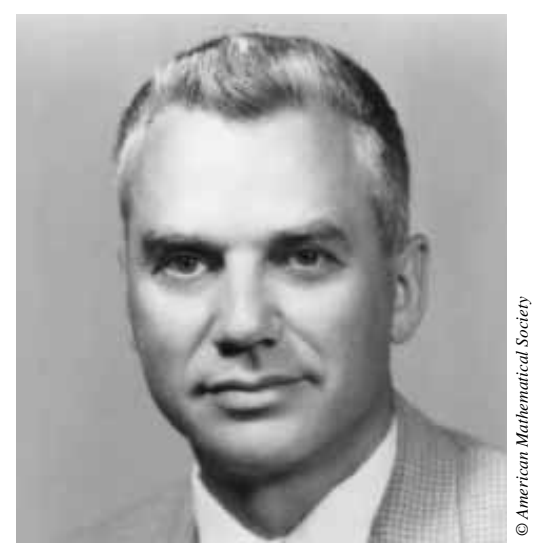

Joseph Leonard Doob, 1910-2004

Joseph Leonard Doob was born in Cincinnati, Ohio, USA on 27 February 1910. In an interview Joe gave for Statistical Science (see Snell (1997)), he said that in grammar school he built a crystal set and in high school operated a radio transmitter. This led him to think he would major in mathematical physics when he entered Harvard in 1926. However, after taking two physics courses, Doob decided to major in mathematics and took three mathematics courses in the first semester of his sophomore year. He graduated with a mathematics major in 1930. Doob remarked:

When I graduated and it was time to think about a Ph.D. degree, I asked Stone (Marshall) to be my advisor. He told me he had no problems for me, that I should go to J. L. Walsh, who always had many problems. Walsh accepted me and we had a fine relationship: he never bothered me, and conversely.

In 1931, Doob received his Master's degree and married Elsie Field. Doob told me he sat next to Elsie at the Boston Symphony for two years before they spoke to each other. He received his $\mathrm{PhD}$ in 1932. His thesis was on boundary values of analytic functions. He published two papers based on his thesis (see Doob (1932), (1933)) in the Transactions of the American Mathematical Society. Doob returned to this subject many years later when he proved a probabilistic version of Fatou's boundary limit theorem for harmonic functions.

Doob applied for and received a two-year National Research Fellowship to work with J. F. Ritt at Columbia University. He chose Columbia because Elsie was a medical student there. He wrote a paper with Ritt, but Doob said that he made only two contributions to this paper: he typed it and he contributed the adjective 'lexicographical' to an order that Ritt had devised.

The Great Depression was still going strong in the 1930s, and Doob could not find a job. B. O. Koopman (also at Columbia) suggested that statistician Harold Hotelling might have a grant that would permit Doob to work with him. Hotelling did, so the Depression led Doob to probability.

In 1933, Kolmogorov provided the first axiomatic foundation for the theory of probability. Thus, a subject that had originated from intuitive ideas suggested by real-life experiences, and 
studied informally, suddenly became mathematics. Probability theory became measure theory with its own problems and terminology: expected value for integral, random variable for function, and event for subset, for example. Doob recognized that this would make it possible to give rigorous proofs for existing probability results, and he felt that the tools of measure theory would lead to new ones. Some felt, and still do, that the emphasis on measure theory is a distraction from probability as applied mathematics. In the interview, Doob commented:

I cannot give a mathematically satisfactory definition of non-mathematical probability. For that matter I cannot give a mathematically satisfactory definition of a non-mathematical chair. The very idea of treating real life as mathematics seems inappropriate to me. But a guiding principle in my work has been the idea that every non-mathematical probabilistic assertion suggests a mathematical counterpart which sharpens the formulation of the nonmathematical assertion and may also have independent mathematical interest.

Doob's approach to probability was evident in his first probability paper (see Doob (1934)). Doob proved theorems related to the law of large numbers, using the fact that the law of large numbers follows from a probabilistic interpretation of Birkhoff's ergodic theorem. He then used these theorems to give rigorous proofs of theorems, proven by Fisher (1922) and Hotelling (1930), related to Fisher's maximum likelihood estimator for estimating a parameter of a distribution.

Fisher defined a consistent estimator as follows:

A statistic satisfies the criterion of consistency, if, when it is calculated from the whole population, it is equal to the required parameter.

Doob interpreted this to mean that the estimates from independent samples of size $n$ converge in probability to the true value. He then showed that, under suitable assumptions, the maximum likelihood estimate is consistent. He also provided conditions such that, for large $n$, the maximum likelihood estimator is approximately normally distributed. These results correspond to those of Hotelling and Fisher, whose proofs Doob said were not rigorous.

This might seem to be a pretty bold claim, given that Fisher was one of the founders of modern statistics and a Cambridge-trained mathematician. But Fisher, referring to his proof of the consistency of the maximum likelihood estimate, wrote:

I am not satisfied as to the mathematical rigor of any proof which I can put forward. For my own part I should gladly have withheld publication until a rigorously complete proof could have been formatted; but the number and variety of the new results which the method discloses press for publication, and at the same time I am not insensible of the advantage which accrues to Applied Mathematics from the cooperation of the Pure Mathematician, and this co-operation is not infrequently called forth by the very imperfections of writers on Applied Mathematics.

In 1935, Doob started his academic career at the University of Illinois. Paul Halmos described Doob's arrival at the University of Illinois Mathematics Department (see Halmos (1985)) as follows:

This boy came in looking like a graduate student, crew cut, shirtsleeves and all. He was 25 years old at the time, I later learned, but he looked 19 or 20. A few days after Doob's arrival my diary starts having many entries such as: 'shoot bull with Doob', and 'Doob's class good'. In one of our early squash games I missed a shot, and swore in exasperation. 
'Just call me Joe', he said. We talked about mathematics, not to the exclusion of politics and music and professional gossip and many other human concerns, but more than about anything else.

The Illinois Mathematics Department was a strong department, but most of the faculty had done their graduate work some years ago. Doob was a breath of fresh air and he quickly acquired outstanding graduate students wanting to work in probability theory. The first three were Paul Halmos, Warren Ambrose, and David Blackwell, all three of whom had been undergraduates at the University of Illinois. I once asked Doob what it was like to start off with such outstanding students. He said 'We were all very young and rather wild'.

Halmos commented that Doob's flippant answers prevented him from being a good teacher. However, I think most people found these flippant answers pretty insightful. Halmos wrote:

Doob has an outgoing, relaxed personality; he seems to get along easily with everyone. He seems to take nothing seriously, but he takes everyone equally seriously.

As an example of this, Doob often remarked that he got his first job on the basis of a false theorem.

I must say that in reviewing Doob's papers, I found them very hard to read. In his paper 'Probability and Doob', Chung (1998) remarked that one often heard that Doob's papers were hard to read, and wrote:

Writing this suddenly put me in mind of my other experience of reading Marcel Proust's interminable recherche of his TIME. Both authors are long-winded, long-drawn-out, and palpably long-suffering, and yet both succeed in making a profound and lasting impact, and prove finalement rewarding. Doob told me that he had read Proust but found him 'boring'. Some readers might say the same of Doob. Proust may be read for his style, no matter the content; Doob should be read for his content, no matter the style.

Doob's students found themselves adopting many of Doob's mannerisms. Doob was aware of this and assured us that these would go away in time. For me this was a long time. It is said that a student of Ambrose met Doob and commented to Ambrose that Doob had adopted many of Ambrose's mannerisms.

Ambrose, writing in Ewing and Gehring (1991), expressed his and Paul's appreciation of Doob in a way that I think most of his students would appreciate:

Another topic that I wish to mention is the appreciation that Paul and I both feel to Joe Doob for what he has taught us, mainly in mathematics but also outside mathematics. Of all the people who have influenced our mathematical and other intellectual development, Joe stands out. Before Doob, neither of us had ever encountered such a forceful thinker. All our previous opinions about the world had to be modified by the serious criticism he made of them.

Doob liked consistency in his life. His car was always a Checker Taxicab. He did not use secretaries and typed (pick-punch) his papers and all seven versions of his famous Stochastic Processes book (see Doob (1953)). For this book, Doob developed a kind of homemade $\mathrm{T}_{\mathrm{E}} \mathrm{X}$, to indicate formulae, Greek letters, etc., which was greatly admired by the printer. 
Doob stayed at the University of Illinois for his entire mathematical career. In the Statistical Science interview, he remarked:

I was charmed by the small-town atmosphere of Urbana as soon as I arrived and never wanted to leave, even though the atmosphere changed through the years.

As an example of this, Doob commented that a neighbor had left some soup for them when he and Elsie moved into their Urbana house.

But perhaps the greatest consistency in Doob's life was his love of fishing in the Illinois rivers and the famous Illinois Hike, which Doob joined in 1939. Here is Doob's description of the hike:

The Saturday Hike was started in 1909 by a classics professor. Each Saturday the group drives to woods along a river. For many years some of the hikers hiked along the river and the others found an open area and played a primitive version of softball baseball, but as the years progressed the numbers dwindled and finally there were too few for baseball. On hot summer days there is swimming in a river or pond.

A 'sitting log' as hiker base is found in the woods. In the evening a fire is built near the log, as large as needed for cooking and warmth, food is cooked, and the problems of the university, Champaign-Urbana and the world are solved. Disagreement on a fact is settled by a Pie for the Hikers bet; the loser brings a pie after the fact has been researched.

The hike went on - rain, snow, or sleet notwithstanding - throughout the year. Doob seldom missed a hike and was the commissar for 25 years. He was not able to go during his last couple of years but grew peppers and other produce for the hike. In return, the hikers provided detailed email descriptions of the hikes, each of which reported a visit to Joe before the hike. In the email of 18 February 2004, we read:

Frank and Sam visited Joe before the hike. We discussed Islam and the renewal theorem.

While Doob enjoyed helping students with their work, he was a bit of a loner when it came to his own mathematics. In the interview, Doob remarked:

I corresponded with many mathematicians but never had detailed interplay with any but Kai Lai Chung and Paul Meyer in probability and Brelot in potential theory. My instincts were to work alone and to collect enough books and reprints so that I could do all my work at home.

One of Doob's best friends was William Feller. This might seem strange, given their very different approaches to probability, but in the interview Doob explained this:

Feller was the first mathematical probabilist I had ever met and, meeting him at a Dartmouth meeting of the AMS around 1940, I felt like Livingston when Stanley found him in Africa.

Feller was writing his classic An Introduction to Probability Theory and Its Applications (see Feller (1950)) at the same time Doob was writing Stochastic Processes. These two books had little in common other than the huge influence that each had in encouraging students to 
work in probability theory. In the interview, Doob commented on one thing they did decide to have in common:

While writing my book I had an argument with Feller. He asserted that everyone said 'random variable' and I asserted that everyone said 'chance variable'. We obviously had to use the same name in our books, so we decided the issue by a stochastic procedure. That is, we tossed for it and he won.

It was common to say that Feller had an analytic approach to probability and Doob a measuretheoretic approach. However, Doob never accepted this distinction. He said he never understood why, when a person is proving something dealing with the distribution of a random variable, he is doing analysis but, when proving something about a random variable using the sample space, he is doing probability.

Doob's first pure probability paper was 'Note on Probability' (Doob (1936)). In this paper, it is assumed that we are offered a sequence of bets with outcomes that are independent and identically distributed random variables with distribution $F$. Doob proves that if we adopt a system for determining which of the bets to make, based only on previous outcomes of the original process, then our fortune will again be independent, identically distributed random variables with the same distribution $F$. This was written well before Doob's interest in martingales.

After this paper, Doob wrote a series of papers to provide the foundation for studying stochastic processes. Kolmogorov defined a probability space as a triple $(\Omega, \mathcal{F}, \mathrm{P})$, where the sample space $\Omega$ is an arbitrary set of points, $\mathcal{F}$ is a $\sigma$-algebra of subsets of $\Omega$ called events, and $\mathrm{P}$ a probability measure defined on $\mathcal{F}$. A random variable $X$ is a measurable function defined on $\Omega$. A stochastic process is a family of random variables $X_{t}(\omega)$ for $t \in T$. If $T$ is a sequence, it is called a discrete-time process and, if $T$ is an interval, it is called a continuous-time process. For fixed $\omega, X_{t}(\omega)$ as a function of $t$ is called the sample path for the process.

The various types of stochastic processes are defined in terms of conditions on their finitedimensional distributions. The Kolmogorov extension theorem guarantees the existence of a stochastic process with a given family of finite-dimensional distributions. For discrete-time processes, the probability measure is unique up to sets of measure 0 . So, for coin tossing, assigning the obvious probability for a finite number of outcomes of the toss of a coin determines a unique measure on the space of infinite sequences, allowing us to prove, for example, that the proportion of heads tends to $\frac{1}{2}$ with probability 1 .

The continuous-time version of coin tossing is Brownian motion. For this process, $T$ is the interval from 0 to infinity. The finite-dimensional distributions are suitable multinomial normal distributions. This process is meant to model a microscopic particle moving randomly in a fluid, so it would be expected to have continuous sample paths. However, the Kolmogorov extension theorem does not ensure that the set of continuous functions will be measurable and, if it is, does not necessarily determine its probability.

For continuous-time stochastic processes, Doob showed that there are many ways to assign a measure with the same finite-dimensional distributions. I recall being very impressed by the following example. Consider a stochastic process that is to represent the position of the hour hand on a clock for all future times. For any fixed time, we assign probability 1 to the event that the hour hand is at 12 . Then, for any finite or denumerable set of times, the hour hand is at 12 for all these times with probability 1 . Of course, this would suggest that the probability that the hour hand is at 12 for all time is 1 . However, Doob showed that there are extensions 
consistent with these finite-dimensional distributions for which the probability that the hour hand is always at 12 is, variously, undefined, 1 , or 0.

Thus, for continuous-time processes we need an additional condition that will assure that the extended measure is more in tune with the finite-dimensional distributions. Doob achieved this by imposing an additional condition, which he called separability. Then he proved that, for any continuous-time process, there is a separable measure having the prescribed finite-dimensional distributions, which will be expected to assign appropriate probabilities to regularity properties of the sample paths. He showed, for example, that if we choose a separable measure for Brownian motion, then the set of continuous sample paths will be measurable and will have probability 1, and, for the Poisson process, the appropriate set of step functions will be measurable and will have probability 1.

I was a graduate student of Doob's during the years 1948-1951. These were busy times for the Doobs. They had three young children, and Elsie was a physician of Internal Medicine at the Carle Clinic in Urbana. The Doobs were generous with their hospitality and I enjoyed many fine meals at their house. At first, I could not understand why Joe and Elsie called each other 'thee' and 'thou' and why Joe always washed the dishes. The 'thee' and 'thou' was explained by the fact that Elsie came from a Quaker family. As for why Doob washed the dishes, I guess he was just ahead of his time.

While Doob was writing Stochastic Processes, Elsie was trying to find her brothers, Noel and Hermann Field. Noel was visiting Prague to gather material for a book when, in May 1949, he mysteriously disappeared. His brother Hermann went to look for him and he also disappeared. Noel had worked for the U.S. State Department (1926-1936), the League of Nations in Geneva (1936-1940), and the Unitarian Service Committee (1942-1946), where he became head of their European office. Hermann had been an architect living in the USA.

Elsie gave up her medical practice to help find her brothers. She eventually got some information about them when Stalin arranged trials to purge certain members of the governments of the communist countries. In their trials, they were charged with having been in contact with Noel or Hermann, who were alleged to have been American spies. Ironically, Noel's name came up in the Hiss trial when ex-Communist Hede Massing testified that she and Hiss had competed in trying to get Noel to join their communist cells. So, in America it was suggested that Noel was a Russian spy.

Elsie went to Washington and pleaded with the government to help get her brothers back, saying that they could then worry about what, if any, kind of spy Noel or Hermann had been. She heard on the radio that Walter Winchell knew something about her brothers, and went to New York to talk to Winchell, only to learn that he knew nothing. Finally, Elsie went to Europe to try to get some information about her brothers.

Before it was all over, Noel's wife Herta and adopted daughter Erika also disappeared. After the death of Stalin, all four were were released, having spent about five years in jail. Hermann had been in a Polish jail, Herta and Noel in separate Hungarian jails, and Erika in a Russian labor camp in the Arctic, where she had been with a railroad-building gang.

In spite of all this, Doob still managed to finish Stochastic Processes. In this book, Doob included his work on the foundations of stochastic processes and discussed the major types - independent, stationary, Markov, Gaussian, uncorrelated, and martingale - providing many new results. Having written this book, Doob predicted that it would never again be possible to include in one book the basic results for stochastic processes.

Doob said the first person he saw reading his book was a student to whom he had given a B grade in his graduate course. The student asked why he got the grade and Doob said it was 
because he felt that he did not seem to understand abstract mathematics. The student replied 'Oh, I thought it was something important'.

While martingales had been studied by Bernstein, Lévy, and Kolmogorov, Doob's interest in martingales came from his review of Ville (1939). Ville did not give a formal definition of the chance processes he called martingales, but he clearly had the idea that it was a process that represented a fair game, and this intrigued Doob. In Doob (1940), he defined martingales, calling them 'processes with property E' - evidently he wanted a more mathematical sounding name than Ville's 'martingale'. He said that property E was suggested as a slightly more modest version of Banach's having called his spaces B-spaces.

In Stochastic Processes, Doob went back to Ville's term 'martingale'. He often commented that he felt that this more colorful name contributed much to the success of martingales; it is not possible to give a talk on martingales without having someone ask for the origin of the name. There are several possible answers, but Ville was undoubtedly thinking of the French gambling system of doubling your bet every time you lose. Thackeray (1853) wrote

'You have not played as yet? Do not do so; above all avoid a martingale, if you do'.

A martingale is also part of a horse's harness. Halmos once sent Doob such a martingale and he had no idea what it was or why he had received it.

A discrete-time martingale is a sequence of random variables with finite expectation such that the expected value of any of the random variables, given the previous outcomes, is equal to the last outcome. Thus, if we interpret the outcomes as our fortune in a game, at each stage the game seems fair. So, we can think of a martingale as representing a fair game. If the expected value is less than or equal to the last outcome, then we say the process is a supermartingale and, if it is greater than or equal to the last value, then it is called a submartingale. Thus, a supermartingale represents an unfavorable game and a submartingale a favorable game. These names are suggested by probabilistic potential theory, where martingales correspond to harmonic functions, supermartingales to superharmonic functions, and submartingales to subharmonic functions.

In his book, Doob called submartingales semimartingales and supermartingales lower semimartingales. He claimed that he realized the connection with potential theory, but that one of his sons was listening to Superman every night and this kept him from using the obvious names. So, he blamed his poor terminology on the fact that he did all his work at home.

Doob proved a number of theorems suggested by the gambling interpretation of these processes. One such theorem is the optional skipping theorem. A player is making a sequence of bets with resulting fortune given by $Y_{1}, Y_{2}, \ldots$ If he skips some bets then his fortune is given by the process $Z_{1}, Z_{2}, \ldots$ defined by $Z_{0}=Y_{0}$ and, for $n>0$, by

$$
Z_{n}=Z_{n-1}+S_{n}\left(Y_{n}-Y_{n-1}\right)=Y_{0}+\sum_{i=1}^{n} S_{i}\left(Y_{i}-Y_{i-1}\right),
$$

where $S_{n}=1$ if he makes the $n$th bet and $S_{n}=0$ if he does not. The decision to play or not to play must depend only on the previous outcomes (no clairvoyance) and it is assumed that the random variables $Z_{n}$ have finite expected values. The optional skipping theorem says that if the $Y$ process is a martingale then so is the $Z$ process, and $\mathrm{E}\left(Z_{n}\right)=\mathrm{E}\left(Y_{n}\right)$; if the $Y$ process is a submartingale then so is the $Z$ process, and $\mathrm{E}\left(Y_{n}\right) \leq \mathrm{E}\left(Z_{n}\right)$; and if the $Y$ process is a supermartingale then so is the $Z$ process, and $\mathrm{E}\left(Y_{n}\right) \geq \mathrm{E}\left(Z_{n}\right)$. Thus, in terms of the player's expected winnings, skipping plays can only hurt him in a favorable game, make no difference in a fair game, and help him in an unfavorable game. 
An important special case of an optional skipping system is an optional stopping system. Here, the player decides to quit betting at some random time $T$ depending only on the outcomes of previous plays. So, for optional stopping, $S_{n}=1$ up to time $T$ and $S_{n}=0$ after this time.

Here is an example of optional skipping. Doob was once asked by a news reporter if he could recommend a strategy for playing roulette. He suggested that the player wait until red comes up once and bet 1 dollar on black, then wait until red comes up twice and bet 1 dollar on black, then wait for red to come up three times and bet 1 dollar on black, etc. Doob claimed that this sounded like a reasonable system of play and the player would not lose very fast by using it.

Here is an example of optional stopping. I was once at a gambling conference and a casino owner claimed that if he took the zeros off the roulette wheel, he would still make a lot of money since most players just play until they lose all their money. Doob's optional stopping theorem for martingales would suggest that he had better leave the zeros on.

Doob kept a card file of ideas for theses. When he got a new graduate student he would pull out a card and suggest the problem on the card. If the student could not solve it, Doob put it back in the file and chose the next card. As a result, we saw future students solving problems that we had not been able to solve.

I succeeded on the third card, which proposed extending to submartingales an inequality called the 'upcrossing inequality' that Doob proved for martingales and used to prove his martingale convergence theorem. This inequality for a submartingale $Y_{1}, Y_{2}, \ldots$ would, for $a<b$, give an upper bound in terms of $\mathrm{E}\left(\left|Y_{n}\right|\right)$ for the expected number of times the sample path can go from below $a$ to above $b$, up to time $n$. This bound implied that if $\mathrm{E}\left(\left|Y_{n}\right|\right)<k$ for some constant $k$, then the sample paths cannot oscillate infinitely often between $a$ and $b$ with positive probability, which implies that the submartingale converges with probability 1.

It turned out to be easy to obtain the desired upper bound by thinking of the submartingale as the price of a stock, and using the optimal skipping theorem to show that a system of buying when the stock gets below $a$ and selling when it gets above $b$, as many times as you can up to time $n$, is not as good as simply buying the stock and holding it for $n$ days. Actually, it turned out to be too easy because Doob said that a thesis had to be at least 30 pages long, so I would have to find something else to pad it with.

Martingales and supermartingales have become important tools in the modern study of mathematical stock market theory. I once asked Doob if he was surprised by the success of martingale theory. His reply was 'No. After all, averaging is a pretty basic concept'. Harmonic functions are also defined by an averaging process. In Kakutani (1944), it was shown that two-dimensional Brownian motion could be used to solve the classical Dirichlet problem, and this started a whole new field of probability relating potential theory and probability theory.

Kakutani came to the USA in 1940 to visit the Institute for Advanced Study for two years. There he met Doob. He and Doob were working together one day with a radio on in the background. It seemed that something important was being announced, so Doob asked Kakutani to put his hand on the antenna. This allowed them to hear that their countries were at war. In 1942, Kakutani had to return to Japan. He wanted to learn probability and, because the Japanese libraries had very few journals, he said that he had had to learn it from Doob's papers in Transactions of the American Mathematical Society! When Kakutani returned to the USA, they became good friends.

Doob then wrote a series of papers showing the connections between potential theory and martingale theory. He became good friends with the famous French potential theorist, Marcel 
Brelot, who was known for his wild driving. Doob introduced him at a conference in France by saying

I never really understood the connection between potential theory and probability theory until I was given a ride in the country by Brelot.

Doob and Brelot played a game in which each would try to prove a result in potential theory that the other could not prove. Doob won by finding a result in classical potential theory suggested by a theorem in the potential theory for Markov processes, whose proof required using the strong Markov property.

Doob suggested to Brelot that they write a book on classical potential theory and probabilistic potential theory, in which Brelot would write the classical theory and Doob the probabilistic theory. As I recall, Brelot wanted to reverse these roles, so Doob ending up writing both himself.

Doob said that he wrote the potential theory book because the success of probabilistic potential theory was leading people to believe that this theory was the whole story. In the Statistical Science interview, he remarked:

I tried to counteract this approach by dealing with classical potential theory first and probability - mostly martingale theory - in later chapters. The result was that even I was surprised to find that classical potential theory and martingale theory were so linked that what at first sight were purely probabilistic notions, such as martingale crossing inequalities, were counterparts of non-probabilistic potential theory, and that proofs in the latter theory gave proofs in the former by the simple device of interpreting.

In fact, not only do you find in this book the counterpart of the upcrossing inequality, but also the counterpart for the second part of my thesis, which involved finding an optimal strategy for playing an arbitrary game. The solution was to stop the first time that the infimum of all supermartingales greater than or equal to the payoff process is equal to the payoff.

In the preface of this 846-page book, Doob thanks his typist, 'usually faithful, sometimes accurate'. He was disappointed that only one person said that he recognized that Doob was thanking himself.

In 1959, Elsie was diagnosed to have cancer. She was given the choice of two years to live if she had radiation treatment, or one year otherwise. She chose the latter and spent the summer with her family at Lake Morey in Vermont, near where my family and I lived. Joe and Elsie showed us many new places for picnics and insisted that we support all the small-town theatrical groups in the area. Joe loved to lecture the restaurants on how much better real Vermont cheese was than the cheese they were serving. Elsie lived until 1991, some 30 years longer than predicted.

After he retired, Joe had not planned to do any more mathematics, but he thought that learning to use a $\mathrm{Mac}^{\circledR}$ and doing some writing would help him adjust to the loss of Elsie. He had given all his books to the University of Illinois, so he had to choose a subject he knew so well that he did not have to refer to any books. He chose measure theory. He had not intended to publish the book, but he could not escape his reputation, and it was published (see Doob (1994)) by Springer.

When Elsie died in 1991, Doob moved to a retirement community, where he adjusted well to his new life. In time he found a wonderful friend, Kay Egbert-Jordan. Kay had a summer place on the lake and Doob renewed his interest in fishing.

I visited Joe a month before he died in 2004. He was still driving and I guess I had the same feeling Joe had while driving with Brelot. However, he was his old self in every other way - 
anxious to talk about politics, his children, the Illinois Hike, his garden, and his latest software for his beloved Mac. In the midst of all this he made one serious comment. He said that he had no desire to live longer for himself but he felt that Kay needed his support.

In the old days, you could type into our main computer 'Edit explain life' and you got the answer 'Life is a supermartingale (i.e. an unfavorable game)'. I tried to think what I would put in for 'Edit explain Doob'. I think my wife Joan suggested the best answer: 'An extraordinarily straightforward guy'.

Dartmouth College, $\mathrm{NH}$

J. LAURIE SNELL

\section{Doob's Honors}

President of the Institute of Mathematical Statistics, 1950.

President of the American Mathematical Society, 1963-1964.

Elected to American Academy of the Arts and Sciences, 1965.

Associate of the French Academy of Sciences, 1975.

Awarded the National Medal of Science by President Carter, 1979.

Awarded the Steele Prize by the American Mathematical Society, 1984.

\section{References}

Chung, K. L. (1998). Probability and Doob. Amer. Math. Monthly 105, 28-35.

Doob, J. L. (1932). The boundary values of analytic functions. Trans. Amer. Math. Soc. 34, 153-170.

Doob, J. L. (1933). The boundary values of analytic functions. II. Trans. Amer. Math. Soc. 35, 418-451.

Doob, J. L. (1934). Probability and statistics. Trans. Amer. Math. Soc. 36, 759-775.

Doob, J. L. (1936). Note on probability. Ann. Math. 37, 363-367.

Doob, J. L. (1940). Regularity properties of certain families of chance variables. Trans. Amer. Math. Soc. 47, $455-486$.

Doob, J. L. (1953). Stochastic Processes. John Wiley, New York.

Dooв, J. L. (1994). Measure Theory (Graduate Texts Math. 143). Springer, New York.

Ewing, J. H. And Gehring, F. W. (eds) (1991). Paul Halmos. Celebrating 50 Years of Mathematics. Springer, New York. Feller, W. (1950). An Introduction to Probability Theory and Its Applications, Vol. 1. John Wiley, New York.

Fisher, R. A. (1922). On the mathematical foundations of theoretical statistics. Phil. Trans. R. Soc. London A 222, 309-368.

Halmos, P. R. (1985). I Want to be a Mathematician. An Automathography. Springer, New York.

Hotelling, H. (1930). The consistency and ultimate distribution of optimum statistics. Trans. Amer. Math. Soc. 32, $847-859$.

KaKUTANi, S. (1944). Two-dimensional Brownian motion and harmonic functions. Proc. Imp. Acad. Tokyo 20, 706-714.

SNELl, J. L. (1997). A conversation with Joe Doob. Statist. Sci. 12, 301-311.

Thackeray, W. M. (1853). The Newcomes: Memoirs of a Most Respectable Family, Vol 1. Bradbury and Evans, London.

VILLE, J. (1939). Etude critique de la notion de collectif. Gauthier-Villars, Paris. 